Yuichiro Kawamura, Tetsuro Imamoto, Junichi Kato, Takashi Haneda, Katsuyuki Tobise, Sokichi Onodera

The First Department of Internal Medicine, Asahikawa Medical College

To determine the site of hypoxic pulmonary vasoconstriciton (HPV), excised canine lung lobes were perfused in the system, consisted of a constant pulsatile pump and an air-chamber, from artery to vein (antegrade perfusion) or vein to artery (retrograde perfusion), and changes in flow amplitude at the inflow site of the lobe during alveolar hypoxia (Hypo) were compared with those of injection of serotonin (S) and histamine (H). Each of them produced significant increases in lobar inflow pressure to the same degree in both perfusional directions, however, flow amplitude changes were different from one another. (S) caused a marked decrease in flow amplitude during antegrade perfusion, and a smajl decrease during retrograde perfusion. (H) caused no change in flow amplitude during antegrade perfusion, but a large decrease during retrograde perfusion. These results indicate that (S) predominantly constrict the arterial side of the pulmonary vascular bed, and $(H)$ selectively constrict the venous side. In contrast with these vasoactive agents, (Hypo) caused a small but significant decrease in flow amplitude during antegrade and retrograde perfusions to the same degree. We conclude that (HPV) occurs not only in the arterial side but in the venous side, and main site of (HPV) located in the peripheral pulmonary vascular bed.

\section{$-343-$ \\ A NEW MEASUREMENT OF PULMONARY VENOUS COMPLICANCE BY R-SYNCHRONIZED VOLUME LOADING}

Toshihisa Asakura, Toshio Mitsui, Jun Miya, Hidehiro Nakajima, Motokazu Hori

Department of Surgery, Institute of Clinical Medicine, University of Tsukuba

Purpose: There is few appropriate method to measure pulmonary venous (PV) compliance (Cpv) due to a difficulty to approach the PV system. we designed a direct and simple measurement of Cpv by R-synchronized volume loading and evaluated the utility experimentally.

Method: In 10 anesthetized open-chest dogs, a balloon (volume $=\Delta V$ ) was inserted in the left atrium through the auricula sinistra. pV pressure wave measured with a low pressure transduser (LPU-0.1-350) indicated $v$ wave during mitral valve closure. Elevated pressure $(\Delta \mathrm{P})$ from control $v$ wave was measured when the balloon $(\Delta V=3-10 \mathrm{ml})$ was inflated during mitral valve closure at the rate of one time per two beats using Model 20-AVCO by a synchronized fashion. using Model $20-A V C O$ by a synchronized fashion.
Results: PV pressure along with volume loading $(3-10 \mathrm{ml})$ was plotted on a regression line with a statistical significance $(r=0.98 \pm 0.02)$. Cpv obtained with our method was concluded to be $0.147 \pm 0.063 \mathrm{ml} \cdot \mathrm{mm} \mathrm{Hg}^{-1} \cdot \mathrm{kg}^{-1}$ when PV pressure was $8.6 \pm 2.3 \mathrm{mmHg}$. The utility of our method is characterized by the prevention of leakage of loading volume to the ventricle and the variableness of loading volume.

Summary:

1) We designed a new method of pulmonary venous compliance measurement by $\mathrm{R}$-synchronized volume loading.

2) Volume-pressure relation $(\Delta V=3-10 \mathrm{ml})$ could be related to a regression line $(r=0.98 \pm 0.02)$. Cpv was concluded to be a static compliance, because it indicated the almost constant value to loading volume change.

3) Cpv obtained with our method was $0.147 \pm 0.063$ $\mathrm{ml} \cdot \mathrm{mmHg}^{-1} \cdot \mathrm{kg}^{-1}$ which was the almost same as proposed in the previous reports.

\section{$-344-$ \\ ESTIMATION OF PULMONARY EXTRAVASCU- LAR WATER VOLUME WITH DOUBLE INDI- CATOR DILUTION METHOD, USING HEAT AND DYE \\ -INFLUENCE OF RESPONSE TIME ON SHAPE OF INDICATOR DILUTION CURVE-}

\author{
Hidetaka Miyazaki, Michio Arakawa, \\ Kenjiro Kambara, Jumpei Iinuma, \\ Takashi Segawa and Senri Hirakawa
}

The Second Department of Internal MediJapan

It has been recognized that the shape of the indicator dilution curve is distorted greatly depending on the time constant $(\tau$, sec) of a detecting system. Since the differences in the mean transit times (MTT) of the thermal-dye dilution curves are required to measure the extravascular lung thermal volume (EVLTV) with the double indicator dilution method, an unavoidable error arises because of the inherent differences in $\tau_{\mathrm{s}}$ between the two detecting systems. Therefore, we recorded response curve of 6 kinds of thermal systems to provide us with thermodilution catheters with properly varying time constants. With the model of a flow loop system perfused with warmed physiological saline solution $\left(37^{\circ} \mathrm{C}\right)$, we recorded two thermodilution curves simultaneously with a pair of thermal systems. Then we assessed the effect of the time constant on the dilution curves in terms of flow $(\dot{Q})$ and MTT. The results were as follows. The calculated flow ( $Q_{\text {TP }}$ ) was not affected by the differences in $\tau_{5}$ and it coincided well with the actual flow in the range of 90-110\%.

In the relations between the differences in the time constants $(\Delta \tau)$ of two detecting systems and the differences in the mean transit times ( $\triangle$ MTT) of those corresponding thermal dilution curves. the following equation was derived,

$\Delta M T T=1.16 \times \Delta \tau-0.03(\Delta \tau, 0.25-1.16 \mathrm{sec})$.

Therefore, it is possible to correct the distortion of the mean transit time produced by the difference in the intrinsic time constant of the detecting system.

In addition, in the animal experiments $(n=12)$ in which the double indicator dilution method was applied with an introduction of the results of the present study and the modified direct destructive method of pearce et al. was perdestructive method of pearce et al. was per-
formed, we confirmed again that about $30 \%$ of EVLTV result from the solid component of the lung and others.

$-345-$

\section{PULMONARY EDEMA DUE TO ACUTE CORO- NARY OCCLUSION IN AWAKE SHEEP}

Toshihiko Toyofuku, Keishi Kubo, Toshishige Shibamoto, Toshio Kobayashi, Shozo Kusama and Gou Ueda\#

The First Department of Internal Medicine, \#The Department of Enviromental Physiology, Shinshu University School of Medicine.

To determine the effects of acute coronary occlusion on pulmonary hemodynamics and lung lymph balance, we investigated three experiments: G-1 